[2] Spinelli FR, Pecani A, et al., BMC Musculosceletal Disord 2017;

[3] Gonzales-Gay MA, et al. Clin and Exp Rheumatol 2006;

[4] Jing Shi, et al. Autoimmun Rev 2014.

Acknowledgements: . EULAR Scientific Bursaries, Programme of 2017

Disclosure of Interest: None declared

DOI: 10.1136/annrheumdis-2018-eular.6217

\section{THU0065 RESULTS OF TREATMENT OF ARTHROSCOPIC AND TRADITIONAL SYNOVECTOMY OF THE KNEE JOINT IN RHEUMATOID ARTHRITIS}

A. Kasimov, I. Khujanazarov. Traumatology, Tashkent Medical Academy, Tashkent, Uzbekistan

Background: Rheumatoid arthritis is a systemic inflammatory autoimmune disease of connective tissue with a predominant joint injury that occurs at any age, more often in women. It is characterised by a chronic course with periods of exacerbations and remissions

Objectives: To evaluate the results of arthroscopic and traditional synovectomy of the knee joint in patients with rheumatoid arthritis

Methods: The study involved 48 patients, 29 of them were women and 19 men, aged from 25 to 65 years old, who had a chronic synovitis of the knee joints. It was implemented at the department of traumatology, orthopaedics and GPH with neurosurgery of Tashkent Medical Academy and 1-Republic Clinical Hospital. In all patients, on the background of basic therapy and intraarticular injections of SCS, a persistent recurrent synovitis was formed within a few months. Sixteen patients underwent sinocapsullectomy (SCE) with the traditional method. 32 patients underwent arthroscopic synovectomy (ASE), for which the standard basic arthroscopic technique Dyonics (Smith and Nephew) was used. The functional condition of the knee joint before and after the operation was assessed using the KOOS scale (Knee and Osteoarthritis Outcome Score)

Results: The study showed that movements in the operated joint were allowed 3-5 hours after the operation, from the first day, isometric exercises were prescribed, active movements in the knee joint before the onset of pain. Results of treatment in terms of up to 3 months were tracked in all 48 patients. The degree of intraarticular changes determined by arthroscopy was evaluated according to the Outerbridge classification. In patients after SCE, pain in the joint, limiting the amplitude of motion, crunching during movement were noted. After ASE, there was a significant improvement in the function and condition of the knee joint: patients could walk for longer distances without additional support, did not experience discomfort while walking the stairs, complaints of pain, and no swelling. Repeated ASE with tunnelling was required in 2 patients with grade III chondromalacia

Conclusions: Thus, the SCE of the knee is indicated if the conservative treatment of recurrent synovitis in RA is unsuccessful for a long time. The ASE should be performed in the early stages of the RA, which drastically reduces the activity of the pathological process, makes it possible to maintain the functional capacity of the joint, and the timely adequate administration of the basic therapy allows the maximum achieved long-term functional result to be maintained for the longest time and reduces the risk of relapse and the need for repeated operations

\section{REFERENCES:}

[1] Steultjens EEMJ, et al. Occupational therapy for rheumatoid arthritis. Cochrane Database of Systematic Reviews 2009.

[2] Sweeney SE, et al. Clinical features of rheumatoid arthritis. In GS Firestein, et al., eds., Kelley's Textbook of Rheumatology 2013(9th ed.);2:1109-1136. Philadelphia: Saunders.

Disclosure of Interest: None declared

DOI: 10.1136/annrheumdis-2018-eular.6999

\section{THU0066 CIRCULATING MIRNAS AS POTENTIAL BIOMARKERS OF DISEASE AND CARDIOVASCULAR RISK IN RHEUMATOID ARTHRITIS PATIENTS}

C. López-Pedrera ${ }^{1}$, N. Barbarroja ${ }^{1}$, P. Ruiz-Limón ${ }^{1}$, S. Remuzgo-Martínez ${ }^{2}$, I. Arias de la Rosa ${ }^{1}$, M.C. Ábalos-Aguilera ${ }^{1}$, Y. Jimenez-Gomez ${ }^{1}$, R. Ortega ${ }^{1}$, E. Collantes ${ }^{1}$, A. Escudero', R. Lopez-Mejías ${ }^{2}$, M.A. Gonzalez-Gay², C. Perez-Sanchez ${ }^{1}$. ${ }^{1}$ IMIBIC/Reina Sofía Hospital/University of Córdoba, Córdoba; ${ }^{2}$ Hospital Universitario Marqués de Valdecilla. IDIVAL. Santander. Universidad de Cantabria, Santander, Spain

Background: Circulating miRNAs have been proposed as attractive candidates as both diagnostic and prognostic biomarkers in various diseases, including a spectrum of autoimmune and cardiovascular conditions. Yet, the contribution of circulating miRNAs to the cardiovascular pathogenesis of Rheumatoid Arthitis (RA) patients and their potential role as biomarkers are still unknown.

Objectives: To identify circulating miRNAs as potential biomarkers of disease features and cardiovascular (CV) risk in RA

Methods: Plasma samples of 48 healthy donors (HDs) and 124 RA patients were collected. In the discovery phase, an array of 2083 human miRNAs was performed by using HTG EdgeSeq miRNA Whole Transcriptome Assay (Next generation sequencing) in 9 plasma samples (3 HDs, and 6 RA patients). Then, differentially expressed miRNAs, were selected and validated by RT-PCR in the whole cohort of patients and HDs. Potential targets of the validated miRNAs were identified by using Ingenuity Pathway Analysis (IPA) software and analysed at protein levels (Multiplex Assay). Correlation and association studies of altered miRNAs with analytical and clinical variables were also performed.

Results: The miRNA whole Transcriptome assay showed that 360 miRNAs were differentially expressed in RA patients in relation to HDs, including 261 upregulated and 97 downregulated. Functional classification (IPA) demonstrated that deregulated miRNAs were mainly involved in processes such as inflammatory response, connective tissue development and function, haematological disease, tissue development, and immunological disease. Nine microRNAs, selected among the most differentially expressed in the array, were selected for validation in all the subjects recruited (miR-299, miR-567, miR-4293, miR-135b, miR-6816, miR-346, miR-143, miR-199a, miR-106a, miR-148b). In silico analyses showed that these miRNAs had potential targets related to cytokine signalling, atherosclerosis pathway and intracellular signalling. The altered levels of selected miRNAs and its putative target proteins were validated in the whole cohort of patients.

A number of serum miRNAs in all the RA patients analysed were found interrelated and associated to autoimmunity (positivity for anti-CCPs and RF), bone erosion, inflammation (CRP, ESR, TNFa, IL6, IL8, IFN and IL-2), and evolution time. We could further identify a specific signature of four miRNAs (miR143, miR106, miR148b and miR567) that identified RA patients that had suffered previous CV events and specifically associated with the increase in the carotid intima-media thickness (CIMT) and with 'Framingham CV risk factors' such as diabetes, obesity or dyslipidemia.

Conclusions: We have branded novel and specific circulating miRNAs related to disease features and CV risk in RA patients, including, their autoimmune and inflammatory profile, the presence of Framingham risk factors and incipient atherosclerosis. These circulating miRNAs might be thus considered useful tools for the management of the disease in this autoimmune condition.

Acknowledgements: Funded by JA (CTS-7940) and the Ministry of Health (ISCIII, PI15/01333 and RIER RD16/0012/0015) cofinanced with FEDER funds.

Disclosure of Interest: None declared

DOI: 10.1136/annrheumdis-2018-eular.6814

\section{THU0067 ASSOCIATION OF HIGH TITERS OF ANTI- CARBAMYLATED PROTEINS ANTIBODIES WITH DECREASED BONE MINERAL DENSITY IN EARLY ARTHRITIS PATIENTS}

I. Arias de la Rosa ${ }^{1}$, A.M. Ortiz ${ }^{2}$, M.D. Boveda ${ }^{1}$, S. Castañeda ${ }^{2}$, I. GonzalezAlvaro', A. Gonzalez' ${ }^{1}{ }^{1}$ Instituto Investigacion Sanitaria- H. Clinico Universitario de Santiago, Santiago de Compostela; ${ }^{2}$ Hospital Universitario La Princesa - ISS-IP, Madrid, Spain

Background: Rheumatoid arthritis (RA) has a negative impact on bone homeostasis, in which multiple inflammatory mediators are involved together with the anti-citrullinated proteins antibodies (ACPA). These antibodies are able to promote osteoclastogenesis and bone loss even before the onset of synovitis Accordingly, ACPA are associated with the three aspects of bone loss: joint bone erosion, juxta-articular bone loss and, as recently demonstrated ${ }^{1}$, decreased bone mineral density (BMD). Other RA specific autoantibodies, the anti-carbamylated protein antibodies (anti-CarPA), are associated with the presence, severity and progression of erosions with independence of ACPA. However, their implication in the other aspects of bone loss has not been studied.

Objectives: To evaluate the association of anti-CarPA with BMD in a cohort of early arthritis (EA) patients.

Methods: Clinical data and samples were obtained at the first visit of 548 patients from the Princesa Early Arthritis Register Longitudinal (PEARL) study, which recruits patients within one year from arthritis onset. BMD was assessed by dualenergy x-ray absorptiometry (Hologic QDR-4500, Elite, Mass, USA) at the lumbar spine (LS), total hip (TH) and metacarpophalangeal (MCP) joints. BMD was considered as Z-scores, except for MCP due to lack of reference data. Anti-CarPA were determined by ELISA using carbamylated fetal calf serum. The ELISA results were considered as negative, below the 98 percentile of healthy controls, low-positive, below the median of positive patients, and high-positive, otherwise Data were analysed with linear regression including sex, age, BMI, and menopause as covariates.

Results: Anti-CarPA were positive in $25.9 \%$ of the EA patients. The positive patients did not show significant differences in BMD with the negative patients. 